\title{
fMRI Using GRAPPA EPI with High Spatial Resolution Improves BOLD Signal Detection at 3T
}

\author{
Dionyssios Mintzopoulos ${ }^{1,2}$, Loukas G. Astrakas ${ }^{1,2}$, Masahiko Hiroki ${ }^{1}$, Azadeh Khanicheh ${ }^{3}$, \\ Lawrence L. Wald ${ }^{1}$, Bruce R. Rosen ${ }^{1}$ and A. Aria Tzika*,1,2
}

\begin{abstract}
${ }^{1}$ Athinoula A. Martinos Center for Biomedical Imaging, Department of Radiology, Massachusetts General Hospital, Harvard Medical School, Charlestown, MA 02129, USA

${ }^{2}$ NMR Surgical Laboratory, Department of Surgery, Massachusetts General Hospital and Shriners Burn Institute, Harvard Medical School, Boston, MA 02114, USA
\end{abstract}

${ }^{3}$ Department of Mechanical and Industrial Engineering, Northeastern University, Boston, MA 02115, USA

\begin{abstract}
We compared GRAPPA parallel MRI (pMRI) to regular MRI (non-GRAPPA) for BOLD fMRI while keeping all other parameters fixed. We acquired both GRAPPA and non-GRAPPA images using a high resolution as well as a low resolution EPI matrix. We found significantly larger values of percent BOLD signal when comparing higher resolution acquisitions to lower resolution ones, independently of whether data were acquired with GRAPPA EPI or with regular EPI. We also demonstrated no loss of functional activation or BOLD signal when comparing GRAPPA to non-GRAPPA while keeping the same spatial resolution. We propose to use pMRI to gain the ability to perform whole-brain acquisition at higher spatial resolution with TE in the 30-40 ms range for optimal BOLD detection at 3T, or at faster scan times. To this end, we conclude that GRAPPA pMRI is advantageous for BOLD fMRI and whole-brain EPI acquisition at high spatial resolution.
\end{abstract}

Keywords: fMRI, EPI, BOLD, GRAPPA, human brain mapping, 3T.

Presented in part at the Meeting of the International Society for Magnetic Resonance 2006, Berlin, Germany

\section{INTRODUCTION}

The underlying principle of functional magnetic resonance imaging (fMRI) is that neuronal activation is accompanied by changes in regional blood flow, volume, and oxygenation [1-7] . An advantage of fMRI is that brain anatomy and activation can be measured simultaneously. The most widely applied fMRI technique for mapping brain activity is Blood Oxygenation Level Dependent (BOLD) imaging, which uses endogenous deoxyhemoglobin as a contrast source [8]. Cortical activation causes alterations in blood oxygenation, which create changes in microscopic susceptibility measured using T2*-weighted sequences [9-11].

Accelerated MRI using parallel magnetic resonance imaging (pMRI) techniques has recently been used in research and clinical procedures in order to improve MRI resolution $[12,13]$. In the context of high magnetic fields, pMRI addresses high-field specific problems such as the need to reduce susceptibility artifacts [14]. Parallel imaging techniques are particularly advantageous in high and ultra high field fMRI, allowing high resolution, high accuracy, and high contrast-to-noise imaging [15]. Advances in both parallel imaging acquisition and reconstruction hold promise for improving the performance of parallel imaging sequences,

*Address correspondence to this author at the Director of NMR Surgical Laboratory, Department of Surgery, Massachusetts General Hospital and Shriners Burn Institute, Harvard Medical School, 51 Blossom Street, Room 261, Boston, MA 02114, USA; Tel: (617) 371-4871; Fax: (617) 720-3544; E-mail: atzika@hms.harvard.edu for reducing artifacts and for increasing spatiotemporal resolution using high acceleration factors [16-18]. The most commonly used parallel imaging techniques relate to $k$-space domain methods (for example, GeneRalized Autocalibrating Partially Parallel Acquisitions, or GRAPPA [13]) or to realspace domain methods (for example, Sensitivity Encoding, or SENSE [12]). SENSE can be more sensitive than GRAPPA to numerical errors or errors in sensitivity profiles while GRAPPA has been found to perform more robustly [19-21] and to have better noise distributional properties [2224]. Overall, the k-space-based reconstruction method of GRAPPA is well suited for fMRI [25]. Indeed, a recent study reported clear superior performance of GRAPPA compared to modified SENSE (mSENSE) in FMRI [26].

pMRI techniques are relatively new and have not been extensively applied in fMRI. pMRI can be used to increase spatial resolution or temporal resolution (i.e. the singlevolume acquisition time, TR). However, because of undersampling, pMRI images have lower signal-to-noise ratio (SNR) and there is concern that detection of the weak BOLD signal may suffer as well. Studies that have employed GRAPPA pMRI for fMRI have reported encouraging results [26-29]. Recently, Preibisch et al. found no significant difference in timeseries Signal-to-Noise Ratio (SNR) when using GRAPPA [26]. Little et al. [28] found that BOLD activations in the occipital cortex were not reduced and for several subjects the detected volume was larger using GRAPPA than without. Lütcke et al. [27] did not detect significant signal difference between non-GRAPPA EPI and 
GRAPPA EPI when the spatial resolution and TE were kept the same in both imaging protocols, but interpreted their results as implying that pMRI is not advantageous for fMRI. Further, Preibisch et al. found that application of SENSE pMRI to fMRI resulted in a substantial increase in speed and/or spatial resolution and in significantly reduced distortions and blurring, while, at the same time, statistical power and the temporal signal to noise ratio (tSNR) of the fMRI timeseries were not affected [29].

In this work, we report on the use of parallel MR methods (GRAPPA) for fMRI of a motor (hand-squeezing) task that has been useful in movement therapy in hemiplegia [30] and in clinical brain mapping of human motor deficits [31]. Using that fMRI protocol and a detailed noise modeling, we investigated the effect of GRAPPA pMRI and higher spatial resolution on the detection of BOLD signal.

\section{MATERIALS AND METHODS}

\section{Study Design}

BOLD fMRI was performed while subjects executed a block-design motor task (hand-squeezing). Each block consisted of $30 \mathrm{~s}$ of squeezing and $30 \mathrm{~s}$ of rest. Each task presentation was comprised of three sequentially presented blocks, resulting in total task duration of $180 \mathrm{~s}$. The arrangement of the acquisitions described below was randomized in order to avoid effects of habituation. Since we employ a block-design paradigm, a TR of $3 \mathrm{~s}$ is adequate; therefore, we chose to focus on optimizing the spatial resolution rather than the temporal resolution (TR) of our experiment in order to exploit fMRI at higher spatial resolution.

Two experiments were performed. In the first experiment, subjects performed the task while four types of images were acquired: GRAPPA EPI images at high spatial resolution, non-GRAPPA EPI (regular EPI) images at high spatial resolution, GRAPPA EPI images at low spatial resolution, and non-GRAPPA EPI images at high spatial resolution. These sets can be arranged, for example, in a $2 \times 2$ matrix with high and low spatial resolution along the two rows and GRAPPA / non-GRAPPA along the two columns. The single-volume acquisition time (TR), echo time (TE), field of view, and number of slices were the same for all images. In particular, TE was long (TE $=42 \mathrm{~ms}$ ) in order to keep the other parameters the same. The in-plane acquisition matrix was the same in the two high spatial resolution images and the same in the two low resolution images, but differed between high and low resolution. Subjects performed the task at the same percentage $(60 \%)$ of their maximum effort level.

In the second experiment, two types of images were acquired: high spatial resolution GRAPPA and low spatial resolution non-GRAPPA. In contrast to the first experiment, the acquisitions in the second experiment are not directly comparable to each other because they have different parameters. Keeping the same parameters for comparison purposes (as was done in the first experiment) prevents the full exploitation of the GRAPPA advantages. Therefore we conducted this second more realistic experiment allowing full optimization of speed and resolution both in the parallel and non-parallel fMRI. This allowed us to test the performance of the two approaches in pulse sequence configurations closer to real clinical applications. To this end, we set $\mathrm{TE}=$
$30 \mathrm{~ms}$, and subjects performed the task at three different percentages $(15 \%, 45 \%$, and $60 \%)$ of their maximum effort level.

Twelve healthy subjects participated in the experiment. Six subjects completed the first experiment, and six subjects completed the second experiment.

All studies were approved by the Institutional Review Board at Massachusetts General Hospital and were performed on a 3T scanner (Siemens TIM Trio) at the Athinoula A. Martinos Center for Biomedical Imaging.

\section{Methods for Hand-Squeezing}

The subjects squeezed the handles of a novel MRcompatible robotic device (MR_CHIROD) [32, 33]. Their maximum squeezing strength was found by adjusting the resistive force necessary to close the handles of the MR_CHIROD until the subjects could barely do so.

The block design paradigm consisted of three alternating action and resting epochs of $30 \mathrm{~s}$ each, lasting a total of 180 s. This design aimed to minimize subject fatigue while still allowing good signal detection. The subjects' squeezing rate was guided by a visually projected metronome stimulus, which projected a cue circle oscillating radially at a frequency of $1 \mathrm{~Hz}$ on a neutral-background screen. A fixation cross was projected during rest. The stimulus was implemented using the Psychophysics Toolbox in Matlab.

Specifically, the task presentation lasted $3 \mathrm{~min} 27 \mathrm{~s}$. The first $27 \mathrm{~s}$ were used for the GRAPPA reference calibration scans (acquired once initially) and to insure the magnetization had equilibrated. The subjects rested during the first 27 $\mathrm{s}$. The magnetization equilibration scans were subsequently discarded, and only the 60 volumes corresponding to the 180 $\mathrm{s}$ were used for analysis.

The subjects' arms were kept extended at their sides and extra padding was used to minimize elbow flexion and further reflexive motion, and to minimize translational and rotational head motion. Typical translational head motion ranged from 0.1-0.4 mm during scans, as reported from the motion correction function in Statistical Parametric Mapping (SPM). All subjects were able to complete the task without difficulty.

Performing the experiment at scaled (percent) levels of each subject's own maximum force of squeezing compensates for performance confounds by constraining betweensubjects performance to make it approximately the same [34]. Subject training typically required approximately 10-15 min, and took place before scanning.

The sequence of acquisitions was randomized in order to avoid effects of habituation.

\section{Methods for Imaging}

\section{First Experiment}

Four imaging protocols were used: high spatial resolution GRAPPA and high spatial resolution non-GRAPPA (regular EPI), and low spatial resolution GRAPPA and low spatial resolution non-GRAPPA (regular EPI). For high spatial resolution whole-brain EPI acquisition for BOLD fMRI, voxel size was $2 \mathrm{~mm} \times 2 \mathrm{~mm} \times 3 \mathrm{~mm}$, (in-plane matrix $96 \times 96$ ) 
$\mathrm{TR} / \mathrm{TE}=3000 / 42 \mathrm{~ms}, 32$ axial slices, $40 \%$ distance factor. Flip Angle $(\mathrm{FA})=90^{\circ}$ at $\mathrm{TR}=3000 \mathrm{~ms}$. The TR/TE $=$ 3000/42 ms were necessary to accommodate both GRAPPA and non-GRAPPA protocols with all other parameters being equal. The high resolution GRAPPA protocol (termed HRG3) had GRAPPA acceleration factor $R=3,92$ GRAPPA autocalibration lines (the maximum allowed number), bandwidth per pixel $754 \mathrm{~Hz} / \mathrm{px}$ (1.43 ms echo spacing). The high resolution non-GRAPPA protocol (HRnG) had bandwidth per pixel $1443 \mathrm{~Hz} / \mathrm{px}$ (0.76 ms echo spacing). The low spatial resolution protocols had 33 slices at $20 \%$ distance factor (the skip factor must be adjusted between high and low resolution protocols to account for the different slice thickness) and $3.1 \mathrm{~mm} \times 3.1 \mathrm{~mm} \times 5 \mathrm{~mm}$ voxel size, which is typical for an fMRI experiment. The GRAPPA protocol (LRG3) had 61 GRAPPA autocalibration lines (the maximum number), $752 \mathrm{~Hz} / \mathrm{px}$ bandwidth. The non-GRAPPA protocol (LRnG) had $988 \mathrm{~Hz} / \mathrm{px}$ bandwidth. GRAPPA EPI reconstruction was done with Sum of Squares (SoS) as implemented by the vendor.

\section{Second Experiment}

In this experiment, GRAPPA was used to reach the highest possible spatial resolution with whole brain acquisition at TE of $30 \mathrm{~ms}$ and TR of $3000 \mathrm{~ms}$. The GRAPPA and nonGRAPPA sequences were not directly comparable. The rationale was to obtain the highest spatiotemporal resolution possible while imaging the whole brain for fMRI, in order to minimize partial-voluming effects that reduce the spatial sensitivity of BOLD fMRI [35-39]. GRAPPA was chosen to implement pMRI in order to reach these goals [19-24]. The number of GRAPPA autocalibration lines (reference lines for GRAPPA calibration) was the maximum possible, in order to ensure the best-quality GRAPPA reconstruction. Single-volume acquisition time TR was the minimum possible at the highest spatial resolution; bandwidth per pixel was minimized to improve signal-to-noise ratio (SNR); and echo time TE was chosen to be $30 \mathrm{~ms}$, following theoretical and empirical arguments suggesting an optimal TE of $30 \mathrm{~ms}$ (with a range for TE from $30 \mathrm{~ms}$ to $40 \mathrm{~ms}$ ) for fMRI at $3 \mathrm{~T}$ $[40,41]$. Typical acquisition parameters for the high spatial resolution GRAPPA protocol were as follows: TR/TE = $3000 \mathrm{~ms} / 30 \mathrm{~ms}$; GRAPPA acceleration factor $R=3$; voxel size of $1.6 \mathrm{~mm} \times 1.6 \mathrm{~mm} \times 3.0 \mathrm{~mm}, 128 \times 128$ in plane matrix, $200 \mathrm{~mm} \times 200 \mathrm{~mm}$ field of view (FOV); 48 slices (5\% skip) covering the entire brain with a tilted axial orientation; 85 GRAPPA autocalibration lines; $1.5 \mathrm{kHz}$ bandwidth per pixel. In comparison, the same spatial resolution without GRAPPA requires sparse fMRI time resolution $(T R=6 \mathrm{~s})$ and very long TE (approximately $60 \mathrm{~ms}$ ) and results in concomitant loss of contrast. GRAPPA EPI reconstruction was done with Sum of Squares (SoS) as implemented by the vendor.

The non-GRAPPA sequence had voxel size of $3.1 \mathrm{~mm} \times$ $3.1 \mathrm{~mm} \times 5.0 \mathrm{~mm}$, and bandwidth per pixel $3.32 \mathrm{kHz}$. TR/TE $=1500 / 30 \mathrm{~ms}$ and a flip angle of $75^{\circ}$ was chosen to maximize gray matter signal at $\mathrm{TR}=1500 \mathrm{~ms}$, assuming that $\mathrm{T} 1$ of gray matter at $3 \mathrm{~T}$ ranges approximately from $1000 \mathrm{~ms}$ to $1300 \mathrm{~ms}$ [42].

\section{Anatomical Reference Scans}

A high resolution 3-dimensional, T1-weighted, MPRAGE (magnetization-prepared rapid gradient echo) image was acquired for anatomical reference and optimal graywhite matter contrast (Sagittal orientation, flip angle $=7^{\circ}$, $\mathrm{TE}=4.73 \mathrm{~ms}, \mathrm{TR}=2530 \mathrm{~ms}$. TI $=1100 \mathrm{~ms}$, voxel resolution $1 \mathrm{~mm}$ isotropic, acquisition matrix $=352 \times 352 \times 192$ ).

\section{Methods for Signal to Noise Ratio (SNR) Measurement}

Both the time-independent spatial SNR (sSNR) and the temporal or timeseries SNR were estimated from unprocessed ("raw") EPI images.

Image, or spatial, SNR (sSNR) was calculated by dividing image intensity values at each voxel, by the (scalar) image standard deviation $S D(x)$ [43]. $S D(x)$ was calculated by fitting the intensity histogram from a noise region of interest (ROI). The noise ROI was drawn near the image edge to contain voxels of the image background. The intensities of the background voxels are assumed to be composites of fluctuations drawn from zero-mean Gaussians. Noise estimated in this manner does not have physiological contributions and is assumed to be additive and solely thermal in origin. GRAPPA sequences had the same TE as their non-GRAPPA counterparts; therefore SNR is proportional to square-root voxel volume and inverse square-root bandwidth per pixel [44] with an additional penalty $R^{1 / 2}$ for GRAPPA. The SSNR metric relies on low-intensity pixels to estimate image noise, but pMRI image unfolding and reconstruction steps suppress the signal outside the brain and introduce spatial correlations that locally alter the noise distribution and can leave residual artifacts near the center of the brain, where coil sensitivities are lower $[45,46]$. Such spatial correlations are not taken into account by this calculation of sSNR. For this reason, in order to avoid the possible pitfalls of sSNR estimation, we proceeded to work with the temporal or timeseries SNR (tSNR).

tSNR includes a component of physiological noise due to breathing, pulsation, and presence of draining vessels [47, 48]. Generally, tNSR will bear the signature of any residual correlations that alter the timecourse of a voxel. tSNR at each voxel is defined from a series of images as the mean (over time) intensity divided by the standard deviation,

$$
t S N R=\langle S(t)\rangle_{t} / S D(t) .
$$

The error model for tSNR hinges on the assumption that voxel intensity is a constant value plus additive random noise. During tSNR calculation, a mask was created from the mean intensity image in order to exclude the voxels outside the brain. The tSNR empirical distributions (histograms) were calculated, normalized to unit area, and fitted by a Gaussian profile. The mean value and standard deviation of the tSNR were estimated from the two Gaussian fit parameters, location $\hat{\mu}$ and full-width-half-maximum $\hat{\sigma}$, of the histograms,

$$
\begin{gathered}
\operatorname{mean}(t S N R)=\hat{\mu} \\
s d(t S N R)=\hat{\sigma}
\end{gathered}
$$


For the purposes of estimating tSNR, unprocessed EPI images were aligned to the first volume in the timeseries. No other processing steps, in particular no preprocessing steps for fMRI, were carried out, i.e. images were not normalized to stereotactic space, and were not smoothed, in order to avoid preprocessing steps that may alter the statistical properties of the timeseries. SPM (SPM2, Wellcome Department of Cognitive Neurology, London, UK, http://www.fil.ion. ucl.ac.uk) was used for image alignment. MRICRO (http://www.sph.sc.edu/comd/rorden/mricro.html) was used for image visualization and presentation. Numerical calculations were performed in Matlab (Version 7.0 R14), The Mathworks, Natick, MA, USA) using custom-written code and standard functions for nonlinear curve fitting. Statistical tests (Shapiro-Wilk for normality, and T-tests or nonparametric Mann-Whitney U-tests for comparison of means) were performed in $\mathrm{R}$ ( $\mathrm{R}$ version 2.6.1, http://www.rproject.org/).

\section{fMRI Analysis}

First-level fMRI analysis was performed with the SPM2 software package (SPM2, Wellcome Department of Cognitive Neurology, London, UK, http://www.fil.ion.ucl.ac.uk). EPI images were corrected for slice-timing, aligned, normal- ized to stereotactic space, reformatted to a standard SPM representation at $2 \mathrm{~mm} \times 2 \mathrm{~mm} \times 2 \mathrm{~mm}$, and smoothed. Activations were determined with $\mathrm{T}$-tests in the whole brain, and threshold was set to $P=0.05$ corrected for multiple comparisons. BOLD signal, or more precisely percent BOLD signal, was calculated from

$$
\text { Percent } \mathrm{BOLD}=100 \times \beta_{1} / \beta_{0}
$$

where $\beta_{0}$ and $\beta_{1}$ are the estimated parameters from the General Linear Model fit to the fMRI timeseries, $\mathbf{Y}=\beta_{0}+\beta_{1} \mathbf{X}$. Activated areas shown in the Results were selected by Boolean conjunction of the BOLD maps with the clusters of voxels that are above threshold $(P<0.05$ corrected) and contain more than 100 voxels per cluster. Images were normalized to stereotactic space and smoothed with a Gaussian kernel of $4 \times$ (voxel dimension). The $4 \times$ kernel was chosen as an empirical optimal choice between maximizing sensitivity through use of large smoothing kernels [49] and retaining ample spatial specificity of the original functional images. A temporal (4 s FWHM) Gaussian filter was applied to account for temporal auto-correlations in the timeseries.

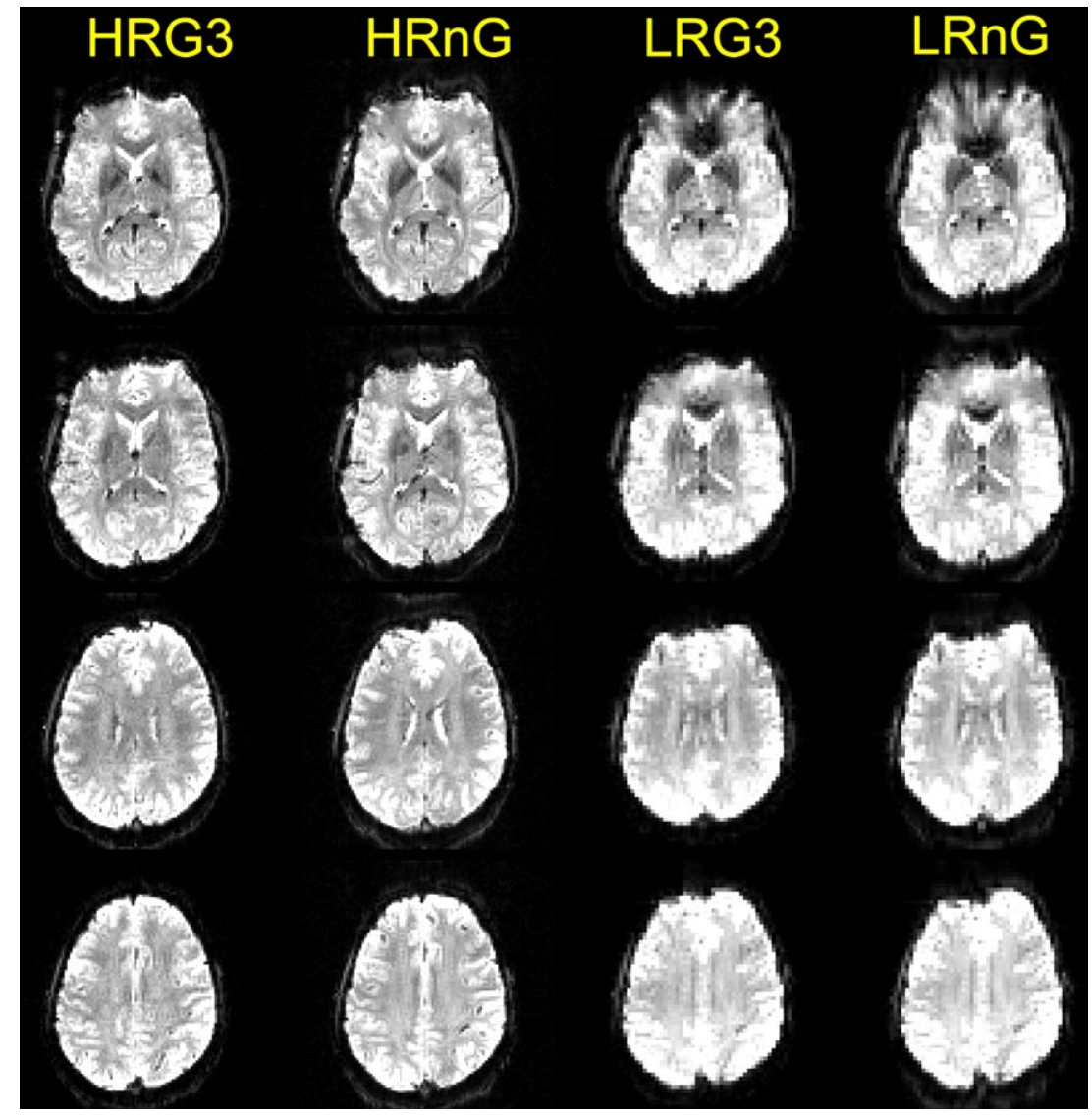

Fig. (1). Comparison of GRAPPA and non-GRAPPA EPI images at high and low resolution. Representative images show the increased blurring and distortions of non-GRAPPA EPI relative to GRAPPA EPI. Four axial slices are arranged from top to bottom rows. Images are from one subject.

Abbreviations: HRG3, high resolution GRAPPA (R=3), HRnG, high resolution non-GRAPPA, LRG3, low resolution GRAPPA (R=3), LRnG, low resolution non-GRAPPA, $(\mathrm{TE}=42 \mathrm{~ms})$. 

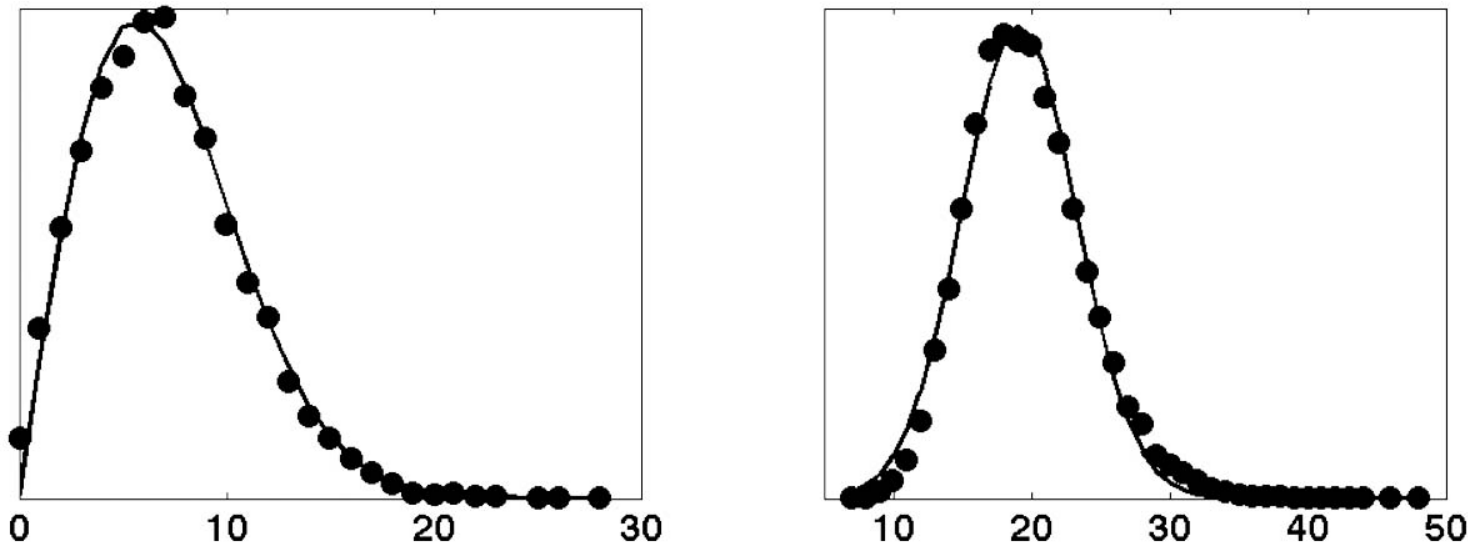

Fig. (2). Typical noise histograms for image SNR (sSNR) calculations. Histograms show the typical distribution of intensities from noise Regions of Interest (ROI) near the edge of the field of view. Left, a non-GRAPPA noise ROI fitted with a Rician distribution. Right, a GRAPPA noise ROI fitted with a non-central Chi-Square distribution.

Abbreviations: HRG3, high resolution GRAPPA ( $\mathrm{R}=3$ ), HRnG, high resolution non-GRAPPA, LRG3, low resolution GRAPPA (R=3), LRnG, low resolution non-GRAPPA.

\section{RESULTS}

\section{First Experiment}

\section{Overall Image Quality}

Typical EPI images (GRAPPA and non GRAPPA, from a single subject), are shown in Fig. (1). Visual inspection shows no apparent artifacts in GRAPPA images. The high resolution images show reduced blurring. GRAPPA images show fewer susceptibility distortions, especially in the frontal areas. This difference in susceptibility distortion persists in the higher slices (bottom row of Fig. 1), although it is not as evident as in the lower slices (top row of Fig. 1).

\section{SNR}

Fig. (2) shows characteristic fits to the noise histograms, used for sSNR calculation. Noise intensities were measured from ROI near the edge of the field of view. The histogram resulting from the non-GRAPPA noise ROI was fit very well by a Rician distribution [50]. The histogram resulting from the GRAPPA noise ROI was fit by a central chi-square distribution [51]. The sSNR metric may be problematic for image analysis (see Methods and Discussion) and was not subsequently employed $[45,46]$.
Summary results for tSNR are listed in Table 1. Representative image-wise tSNR calculations from a single subject are shown in Fig. (3), depicting the mean intensity and standard deviation images calculated from raw EPI images, the tSNR image, and the tSNR empirical distribution (histograms). The tSNR histograms are fitted very well by Gaussian curves (fourth row of Fig. 3). The tSNR mean value and standard deviation were estimated distributionally from the tSNR histogram. Representative fit curves are shown superimposed on the tSNR histograms (fourth row of Fig. 3) and also plotted together (Fig. 4). Values for tSNR are listed in Table 1. The values for tSNR for the high resolution nonGRAPPA (HRnG) images are slightly higher compared to GRAPPA (HRG3) (Table 1 and bottom two panels of Fig. 6). The difference reached statistical significance with paired T-test uncorrected for multiple comparisons (Table 2) and also when the Bonferroni correction for multiple comparisons is employed (Table 3). There was no statistically significant difference in tSNR between the low resolution LRG3 and LRnG images, nor between HRG3 and low resolution acquisitions (LRG3, LRnG) (bottom two panels of Fig. 6).

Table 1. Summary Statistics from fMRI Activated Clusters in the Motor/Somatosensory Region (First Experiment)

\begin{tabular}{|c|c|c|c|c|}
\hline Maximum percent BOLD & $5 \pm 1$ & $4.6 \pm 0.6$ & $3.5 \pm 0.7$ & $3.3 \pm 0.7$ \\
\hline Number of activated voxels ${ }^{\mathrm{a}}$ & $2600 \pm 1000$ & $2600 \pm 1200$ & $3500 \pm 1700$ & $5000 \pm 3000$ \\
\hline Number of activated voxels ${ }^{\mathrm{b}}$ & 1549 & 1350 & 1027 & 2583 \\
\hline tSNR & $16 \pm 4$ & $20 \pm 5$ & $16 \pm 3$ & $16 \pm 2$ \\
\hline
\end{tabular}

${ }^{\text {a }}$ Summary Statistics from first-level data.

${ }^{\mathrm{b}}$ Second-level results (group activation, random effects).

Abbreviations: HRG3, high spatial resolution GRAPPA (R=3), HRnG, high spatial resolution non-GRAPPA, LRG3, low spatial resolution GRAPPA (R=3), LRnG, low spatial resolution non-GRAPPA. 


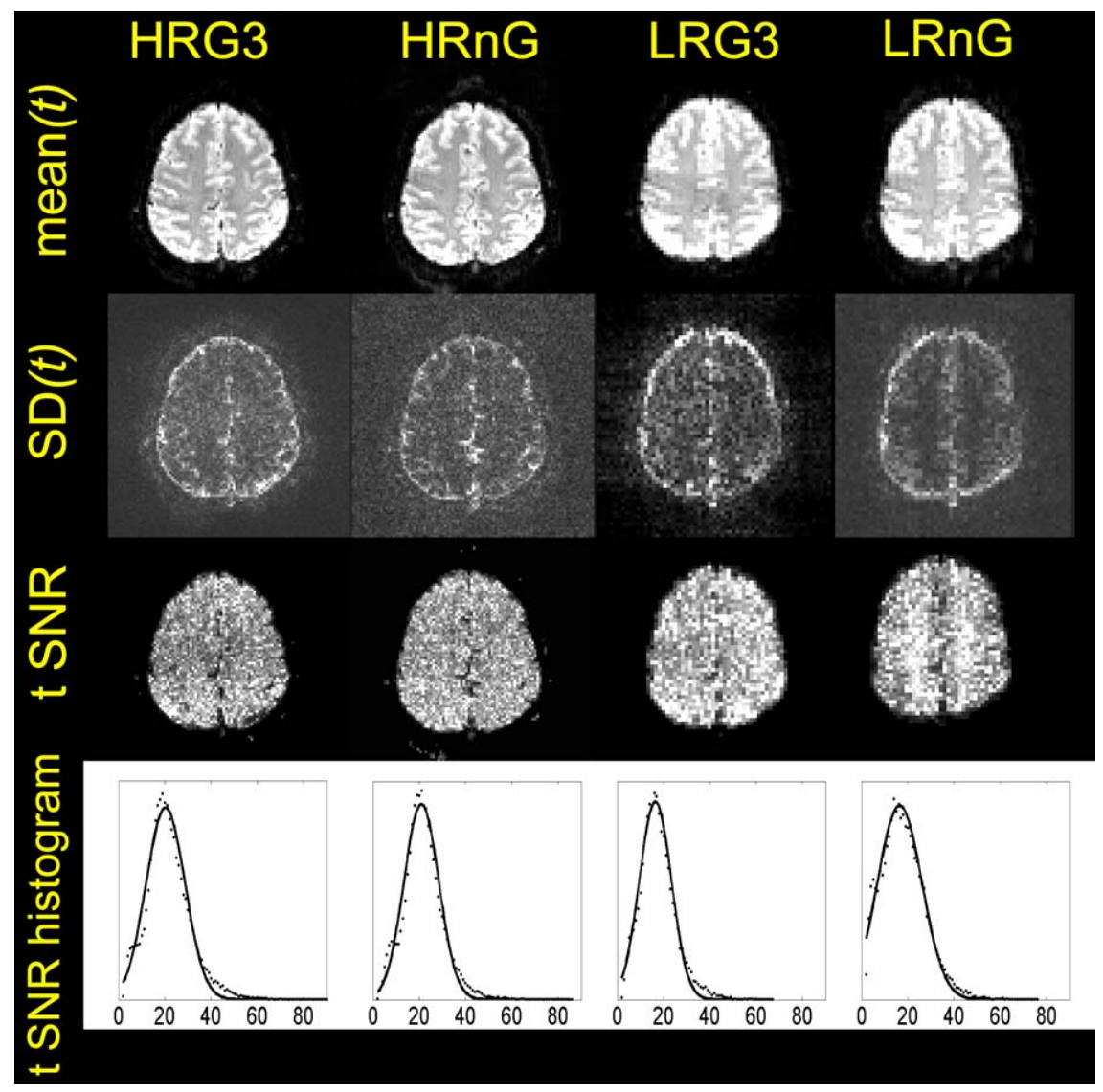

Fig. (3). Typical timeseries SNR (tSNR) calculations and results. Top row depicts the mean intensity image computed from an epoch of EPI images from each of the four protocols, HRG3, HRnG, LRG3, and LRnG. The second row depicts the standard deviation (SD $(t))$ of the timeseries. The third row shows the timeseries SNR (tSNR) computed from the first and second rows, mean and SD images. The fourth row shows the distribution of image intensities of the tSNR images. In each case, the histograms were normalized to unit area and fitted with a Gaussian. These Gaussian fit distributions are shown together in Fig. (4). Images are from a single subject.

Abbreviations: HRG3, high resolution GRAPPA ( $\mathrm{R}=3$ ), HRnG, high resolution non-GRAPPA, LRG3, low resolution GRAPPA (R=3), LRnG, low resolution non-GRAPPA.

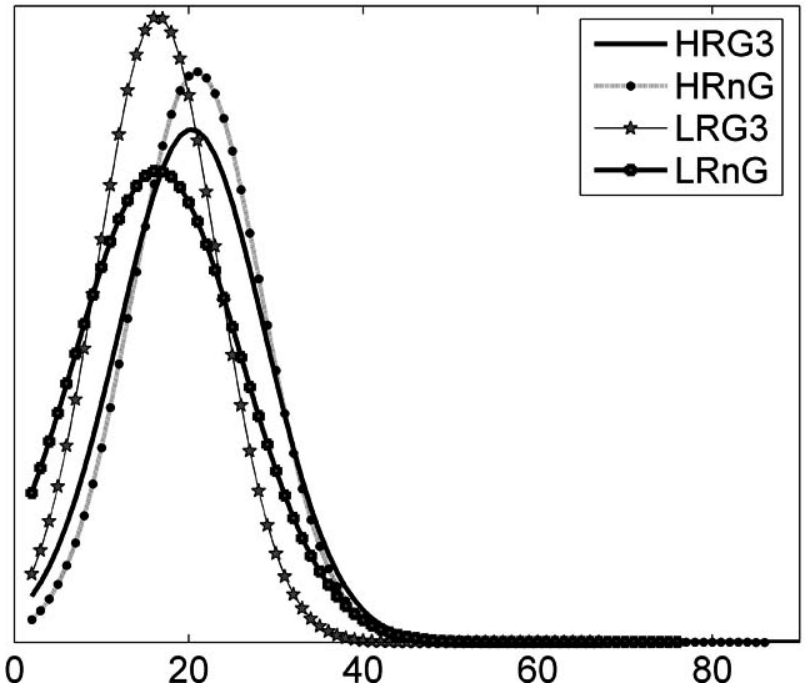

Fig. (4). Comparison of typical tSNR intensity distributions. The four Gaussian fit distributions of Fig. (3) are shown superimposed together. Each distribution is normalized to unit area.

Abbreviations: HRG3, high resolution GRAPPA ( $\mathrm{R}=3$ ), HRnG, high resolution non-GRAPPA, LRG3, low resolution GRAPPA $(\mathrm{R}=3), \mathrm{LRnG}$, low resolution non-GRAPPA.
We compared the ratios of tSNR of GRAPPA to tSNR of non-GRAPPA versus theoretical ratios calculated assuming thermal noise [44]. We first compared GRAPPA to nonGRAPPA at the same resolution (HRG3 to HRnG and LRG3 to $\mathrm{LRnG})$. In that case, theoretical SNR is inversely proportional to the square root of bandwidth per pixel [44] with an additional penalty $R^{1 / 2}$ for GRAPPA, with all other parameters (TE, field of view and acquisition matrix, and number of averages) being equal. Using the values of bandwidth per pixel from Methods and acceleration factor $R=3$ gives the twin theoretical estimates SNR(HRG3)/SNR(HRnG) $=0.800$ and $\mathrm{SNR}(\mathrm{LRG} 3) / \mathrm{SNR}(\mathrm{LRnG})=0.662$. The experimental ratio at high resolution is $\mathrm{tSNR}(\mathrm{HRG} 3) / \mathrm{tSNR}(\mathrm{HRnG})=0.8$ \pm 0.2. The low resolution experimental ratio is $\mathrm{tSNR}(\mathrm{LRG} 3) / \mathrm{tSNR}(\mathrm{LRnG})=1.0 \pm 0.1$. We then compared high resolution acquisitions to low resolution ones. In this case, theoretical SNR (assuming thermal noise) scales in proportion to the square root of voxel volume and in inverse proportion to the bandwidth for thermal noise. The acceleration factor does not influence the calculation of the theoretical ratios since it is the same for both high and low resolution EPI protocols. The theoretical ratios are $\mathrm{SNR}(\mathrm{HRG} 3) / \mathrm{SNR}(\mathrm{LRG} 3)=0.52, \mathrm{SNR}(\mathrm{HRnG}) / \mathrm{SNR}(\mathrm{LRnG})$ 


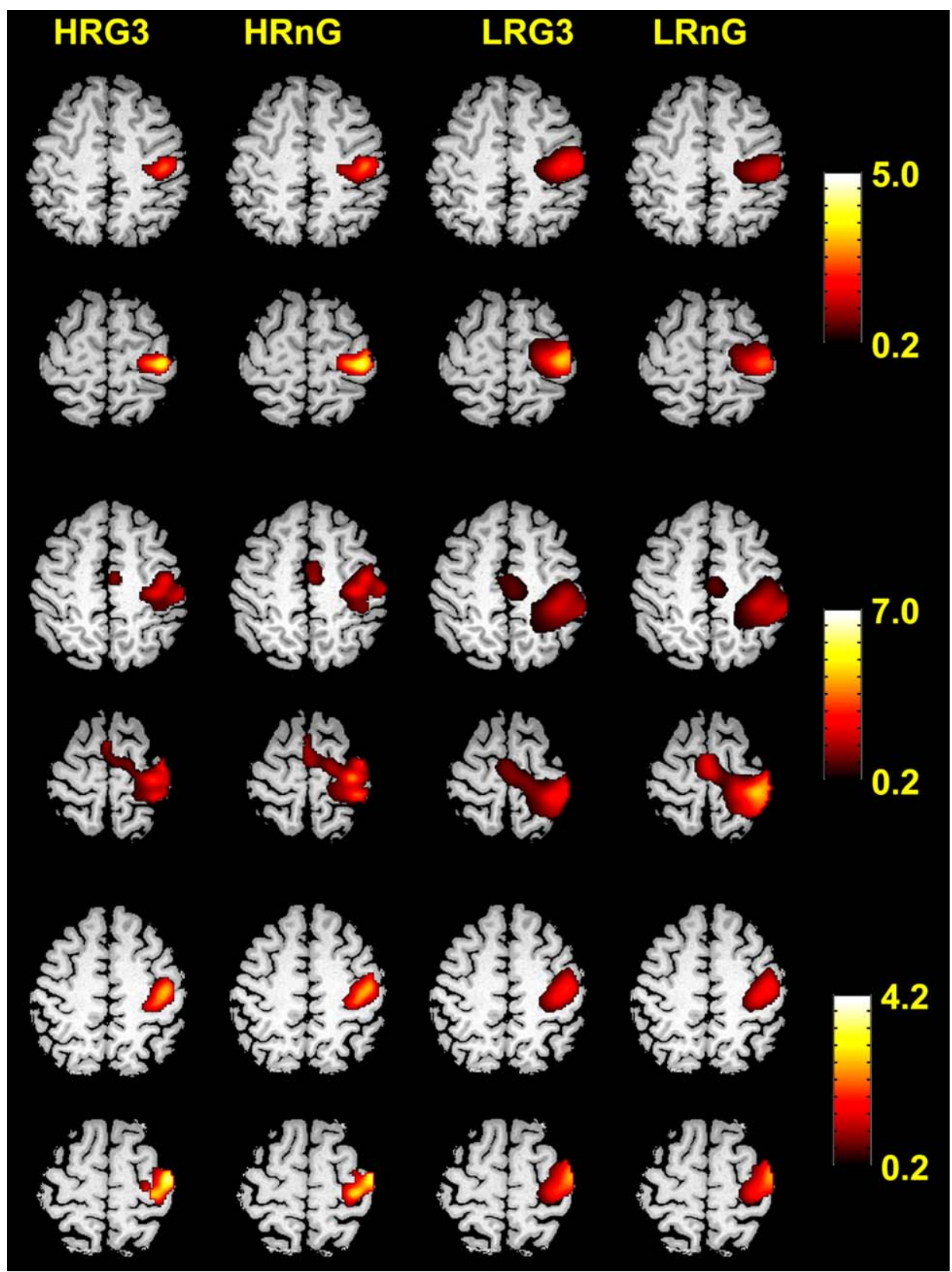

Fig. (5). Typical BOLD signal values and spatial activation profiles for each of the four acquisition protocols. Images are shown from three subjects (two axial slices from each subject at the level of the motor and somatosensory cortex). Subject 1, top two rows; subject 2, third and fourth rows; subject 3, fifth and sixth rows. The BOLD signal is more intense in the high resolution (HRG3, HRnG) than in the low resolution (LRG3, LRnG) images (BOLD is scaled to the same range). There is no essential difference in activated area between the GRAPPA and non-GRAPPA images (HRG3 compared to HRnG and LRG3 compared to LRnG). The low resolution images show more activation volume, but they are more blurred; the high resolution images show better cortical specificity than the low resolution images. The activated areas encompass the voxels that were significant at $\mathrm{P}<0.05$, corrected.

Abbreviations: HRG3, high resolution GRAPPA ( $\mathrm{R}=3$ ), HRnG, high resolution non-GRAPPA, LRG3, low resolution GRAPPA (R=3), LRnG, low resolution non-GRAPPA.

$=0.43$, and $\mathrm{SNR}(\mathrm{HRG} 3) / \mathrm{SNR}(\mathrm{LRnG})=0.30$. All experimentally calculated ratios break this scaling relationship. Specifically, $\mathrm{tSNR}(\mathrm{HRG} 3) / \mathrm{tSNR}(\mathrm{LRG} 3)=1.0 \pm 0.2$, $\mathrm{tSNR}(\mathrm{HRnG}) / \mathrm{tSNR}(\mathrm{LRnG})=1.2 \pm 0.2$ and $\mathrm{tSNR}(\mathrm{HRG} 3) /$ $\operatorname{tSNR}(\mathrm{LRnG})=1.0 \pm 0.3$.

\section{fMRI - Volume of Activation}

While the low resolution acquisitions resulted in larger activation volume (Table $\mathbf{1}$ ), the increase barely reached statistical significance with paired T-tests uncorrected for 

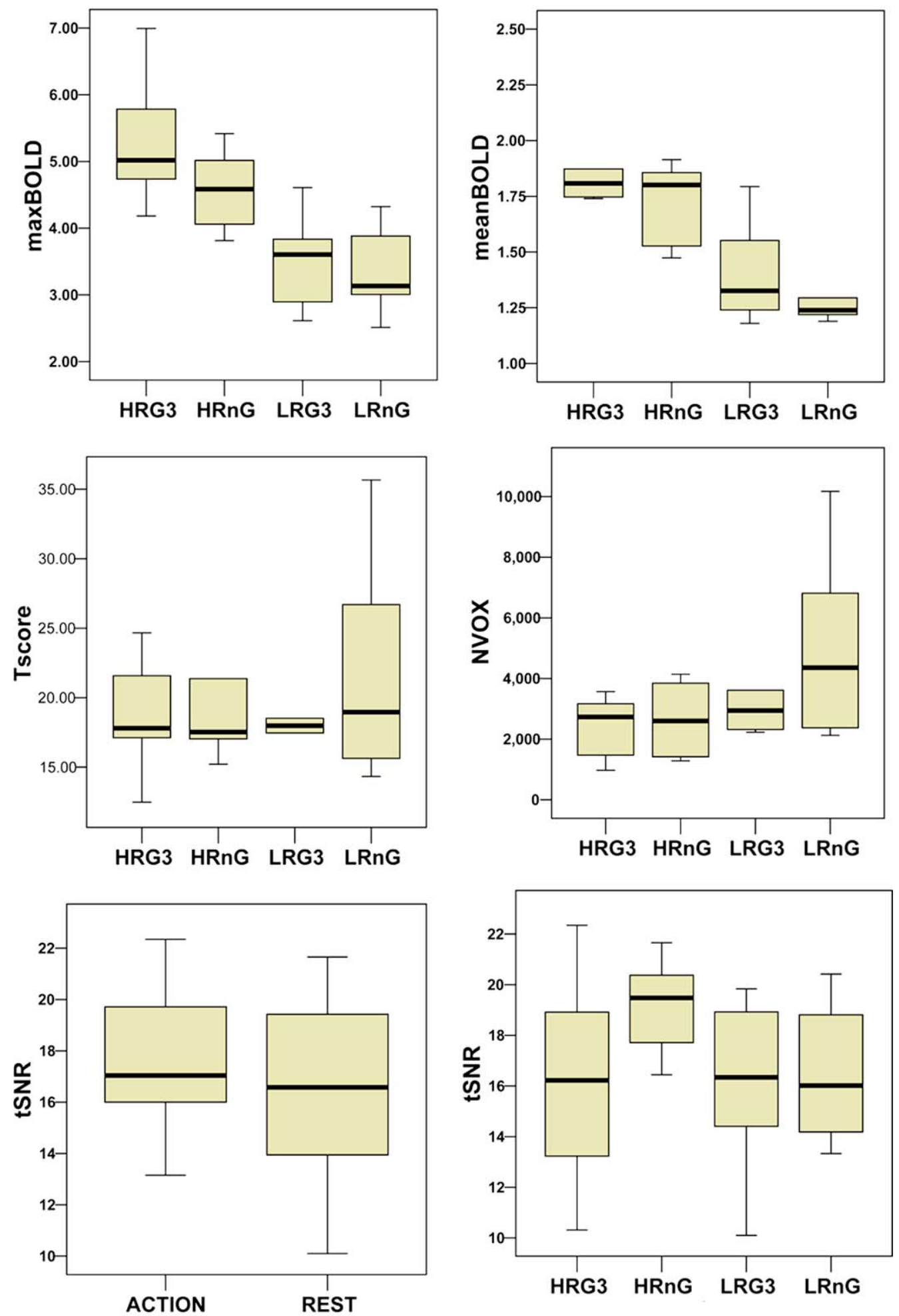

Fig. (6). Comparison of summary results. The box plots show the distributions of the maximal percent BOLD (maxBOLD), the mean percent BOLD (meanBOLD), the maximal T-scores (Tscore), the number of activated voxels (NVOX) and the tSNR. The two tSNR box plots are arranged in the following manner, one with the action / rest epochs as factor and the second with acquisition as factor.

Abbreviations: HRG3, high resolution GRAPPA $(\mathrm{R}=3)$, HRnG, high resolution non-GRAPPA, LRG3, low resolution GRAPPA (R=3), LRnG, low resolution non-GRAPPA.

multiple comparisons (Table 2) and did not reach statistical significance after correction for multiple comparisons (Table 3). The dispersion of the activated volumes at low resolution was larger than the dispersion of the high resolution acquisitions (Table 1 and Fig. 6). Fig. (7) shows group activation using image-wise second-level statistics (random effects, 
Table 2. Comparison between High and Low Spatial Resolution Images and between GRAPPA and Non-GRAPPA ${ }^{\mathrm{a}}$

\begin{tabular}{|c|c|c|c|c|}
\hline & & HRnG & LRG3 & LRnG \\
\hline \multirow[t]{2}{*}{ Number of activated voxels } & $\mathrm{HRnG}$ & & 0.2454 & 0.0646 \\
\hline & LRG3 & & & 0.2590 \\
\hline \multirow{2}{*}{ Maximum T-score } & HRG3 & 0.4555 & 0.6517 & 0.3031 \\
\hline & LRG3 & & & 0.3929 \\
\hline \multirow{3}{*}{ tSNR } & HRG3 & $0.0106 *$ & 0.7889 & 0.8144 \\
\hline & HRnG & & $0.0146 *$ & $0.0085^{*}$ \\
\hline & LRG3 & & & 0.9402 \\
\hline \multirow{3}{*}{ Mean percent BOLD } & HRG3 & 0.0951 & $0.0083 *$ & $0.0018^{*}$ \\
\hline & HRnG & & $0.0357^{*}$ & $0.0020^{*}$ \\
\hline & LRG3 & & & 0.4402 \\
\hline
\end{tabular}

${ }^{\mathrm{a}} \mathrm{P}$-Values, Paired T-test.

* Statistically significant.

Abbreviations: HRG3, high spatial resolution GRAPPA ( $\mathrm{R}=3$ ), HRnG, high spatial resolution non-GRAPPA, LRG3, low spatial resolution GRAPPA (R=3), LRnG, low spatial resolution non-GRAPPA.

Table 3. Comparison between High and Low Spatial Resolution Images and between GRAPPA and Non-GRAPPA ${ }^{\mathrm{a}}$

\begin{tabular}{|c|c|c|c|c|}
\hline & & HRnG & LRG3 & LRnG \\
\hline \multirow{3}{*}{ Number of activated voxels } & HRG3 & 1.0000 & 1.000 & 0.2032 \\
\hline & HRnG & & 1.0000 & 0.2940 \\
\hline & LRG3 & & & 1.0000 \\
\hline \multirow{3}{*}{ Maximum T-score } & HRG3 & 1.0000 & 1.0000 & 1.0000 \\
\hline & HRnG & & 1.0000 & 1.0000 \\
\hline & LRG3 & & & 1.0000 \\
\hline \multirow{3}{*}{ tSNR } & HRG3 & $0.0351 *$ & 1.0000 & 1.0000 \\
\hline & HRnG & & 0.0640 & 0.0607 \\
\hline & LRG3 & & & 1.0000 \\
\hline \multirow{3}{*}{ Maximum percent BOLD } & HRG3 & 0.7164 & $0.0038 *$ & $0.0013 *$ \\
\hline & $\mathrm{HRnG}$ & & 0.1528 & 0.0578 \\
\hline & LRG3 & & & 1.0000 \\
\hline \multirow{3}{*}{ Mean percent BOLD } & HRG3 & 0.7054 & $0.0022 *$ & $0.0002 *$ \\
\hline & $\mathrm{HRnG}$ & & $0.0307 *$ & $0.0030 *$ \\
\hline & LRG3 & & & 1.0000 \\
\hline
\end{tabular}

${ }^{\mathrm{a} P}$-Values, ANOVA with Bonferroni Correction for Multiple Comparisons.

* Statistically significant.

Abbreviations: HRG3, high spatial resolution GRAPPA (R=3), HRnG, high spatial resolution non-GRAPPA, LRG3, low spatial resolution GRAPPA (R=3), LRnG, low spatial resolution non-GRAPPA. 


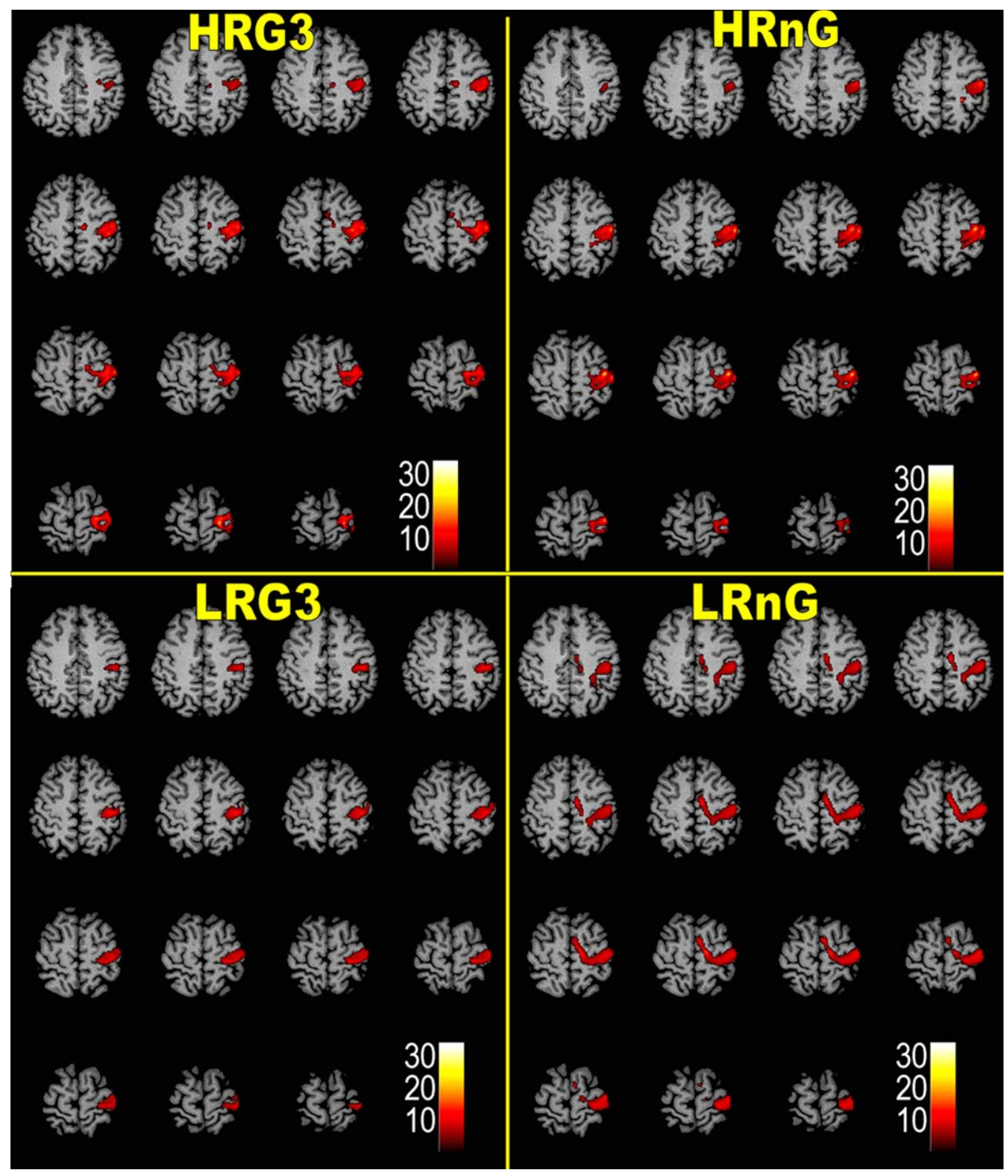

Fig. (7). Comparison of group activation patterns. The image shows group activations for each type of acquisition. The high resolution acquisitions show more detailed activation patterns in the cortical area, and the high resolution GRAPPA also shows activation in the supplementary motor area ( $\mathrm{N}=6$, random effects, $\mathrm{P}<0.001$ uncorrected). (Group comparison, color denotes statistical significance ( $\mathrm{T}$-scores)). Abbreviations: HRG3, high resolution GRAPPA $(\mathrm{R}=3)$, HRnG, high resolution non-GRAPPA, LRG3, low resolution GRAPPA (R=3), LRnG, low resolution non-GRAPPA.

$\mathrm{P}<0.001$, uncorrected). The group activation provides a measure of robustness of the measurements. The group activation in the low resolution is not significantly larger compared to the high resolution. The activated somatosensory cortex volume for LRG3 is approximately the same as that for the HR (HRG3, HRnG) values. The LRnG activated volume is larger, but the activation seems to connect the somatosensory area to the supplementary motor area (Fig. 7) and may be partly spurious.

\section{fMRI - T-Scores}

The variation in T-scores from the activated area in the contralateral somatosensory cortex was small (Tables 2 and
3) and statistical comparison of the T-scores themselves did not reveal any statistical significance (P-values shown in Tables $\mathbf{2}$ and 3). Specifically, the high resolution acquisition T-scores were compared to the low resolution T-scores (HRG3 compared to LRG3 and HRnG compared to LRnG), as well as comparison between the GRAPPA and the nonGRAPPA T-scores (for example, HRG3 compared to HRnG and LRG3 compared to LRnG).

\section{$f M R I-B O L D$}

Table 1 shows that the BOLD signal is approximately the same in GRAPPA compared to non-GRAPPA when spatial resolution is held constant. This holds true for both the 
Table 4. Mean Percent BOLD Signal Detected with High Resolution GRAPPA EPI fMRI Compared to Low Resolution NonGRAPPA EPI IMRI ${ }^{a}$

\begin{tabular}{|c|c|c|c|c|}
\hline Effort Level $^{\text {b }}$ & $\begin{array}{c}\text { BOLD, high resolution } \\
\text { GRAPPA }^{c}\end{array}$ & $\begin{array}{l}\text { BOLD, low resolution non- } \\
\text { GRAPPA }^{c}\end{array}$ & $P$-Value ${ }^{\mathrm{d}}$ & $\begin{array}{l}\text { Ratio of high resolution GRAPPA to } \\
\text { low resolution non-GRAPPA }\end{array}$ \\
\hline $15 \%$ & $1.7 \pm 0.5$ & $0.7 \pm 0.3$ & 0.0079 & $2.4 \pm 0.5$ \\
\hline $45 \%$ & $1.8 \pm 0.6$ & $0.9 \pm 0.3$ & 0.0305 & $2.0 \pm 0.5$ \\
\hline $60 \%$ & $1.8 \pm 0.7$ & $0.6 \pm 0.3$ & 0.0079 & $3.1 \pm 0.8$ \\
\hline
\end{tabular}

${ }^{\mathrm{a}}$ Second experiment, TE $=30 \mathrm{~ms}$.

${ }^{b}$ Effort measured as percentage of each subject's own maximum.

${ }^{c}$ Mean $\pm \mathrm{SD}(\mathrm{N}=6)$

${ }^{\mathrm{d}}$ T-test, two-tailed.

${ }^{\mathrm{e}}$ Ratio of mean BOLD values $\pm \mathrm{SD}$

maximal value (peak value from the activated area in the contralateral somatosensory cortex) of percent BOLD as well as for the mean percent BOLD from the activated area in the contralateral somatosensory cortex (Table 1). Representative results for BOLD values from three of the six subjects are shown in Fig. (5). These results are further visualized in Fig. (6), which shows summary results from the first-level measurements (maxBOLD denotes the maximal percent BOLD values and meanBOLD the mean percent BOLD values).

The difference between high and low resolution BOLD values is significant and robust under statistical testing. The comparison between HRnG and LRG3 is highly significant when a paired T-test is used (Table 2) and is still significant but less so with $\mathrm{P}$-value $\mathrm{P}=0.021$ when a simple $\mathrm{T}$-test is used. It was not significant under multiple comparisons with Bonferroni correction (Table 3 ). The comparisons between HRG3 and LR (LRG3, LRnG) retain high statistical significance both with paired T-test and under multiple comparison correction (Tables 2 and 3), while the HRnG - LRnG comparison is close to statistical significance $(\mathrm{P}=0.0578)$ under correction for multiple comparisons (Table $\mathbf{3}$ ).

The difference in measured BOLD was also statistically significant in the second experiment (Table 4), where the subjects performed the experiment at various effort levels.

\section{Second Experiment}

Fig. (8) shows that activation was detected in the same brain areas and followed the same pattern using both the GRAPPA and non-GRAPPA EPI protocols.

Measured percent BOLD signal changes were consistently larger using the high resolution GRAPPA protocol (Table 4). This finding remained true as subjects performed the task across three different effort levels (levels of squeezing force). Table 4 compares group-average BOLD signal in the non-GRAPPA low resolution and in the GRAPPA high resolution protocols. The measured BOLD ratios (high resolution divided by low resolution) were averaged over the number of subjects, and the average figures were compared at each squeezing level using two-tailed T-tests. The increase in signal was found to be significant in all cases $(P$-values listed in Table 4). The ratio of activated volumes (low resolution non-GRAPPA to high resolution GRAPPA) was close to one, and the grand average over subjects and effort levels was $0.8 \pm 0.4$. The ratios of activated volumes in this case did not significantly differ from $1(P=0.2330$, T-test, twotailed).

\section{DISCUSSION}

The principal finding of this study is that a high resolution imaging protocol resulted in improved detection of BOLD signal, measured by higher values of percent BOLD
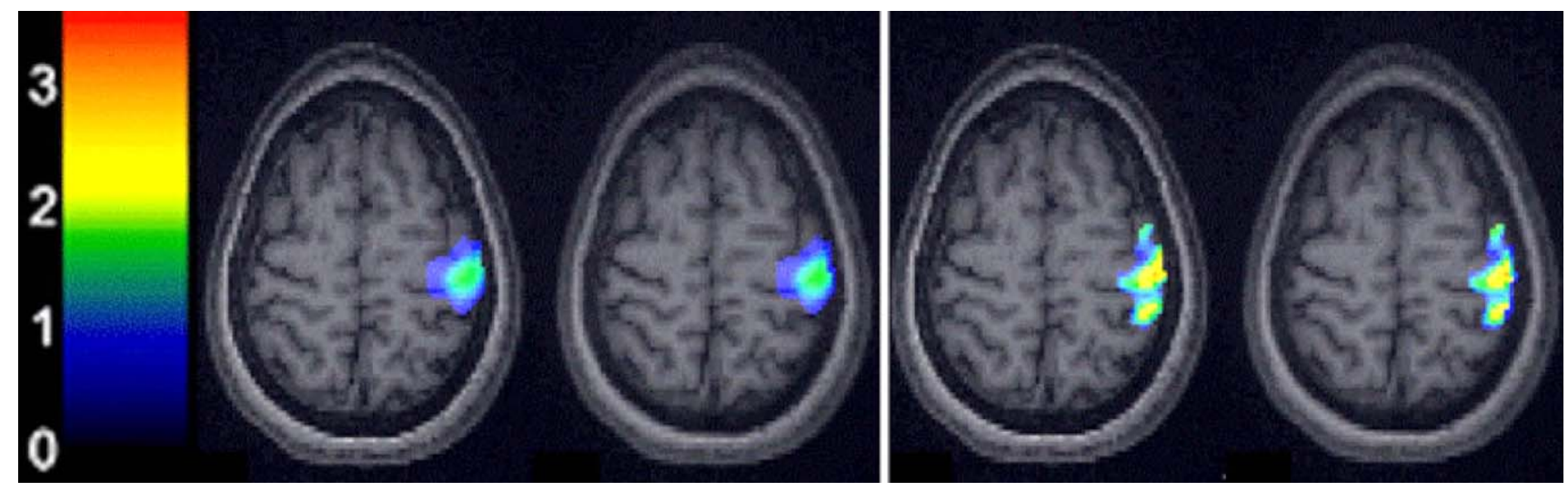

Fig. (8). Comparison of activation profiles and BOLD signal values between a high resolution GRAPPA fMRI experiment and a low resolution fMRI experiment. BOLD signal is higher from the high resolution GRAPPA experiment, and the spatial activation profile shows much better specificity. (Second experiment). 
signal, with respect to a low resolution imaging protocol. In addition, we found that using GRAPPA is not detrimental to fMRI. Thus, comparison of GRAPPA to non-GRAPPA keeping all other parameters the same did not result in any significant loss of calculated BOLD signal and/or activation volume. However, the comparison of high resolution to low resolution resulted in statistical significance that was found to be robust under statistical testing, as it was true not only for comparisons with T-tests but also for comparisons corrected for multiple comparisons (Tables $2, \mathbf{3}, \mathbf{6}$, and Fig. 6). Thus, our results support our conclusion that the significant improvement in BOLD detection observed herein is a true effect and not a random one (BOLD is a physiological measure and should be independent of the means of detection, in as far as the ratio $\beta_{1} / \beta_{0}$ removes any multiplicative factors); and that measurement of the physiological effect (BOLD) is not affected by use of GRAPPA. Instead, measurement of BOLD is improved through the use of GRAPPA pMRI to perform fMRI at higher spatial resolution.

Calculation of other metrics demonstrated that performing fMRI at higher spatial resolution and using GRAPPA for fMRI was not detrimental to the quality of the data. Although the activated volumes were larger with the low resolution acquisitions, so was the dispersion of those results (Table $\mathbf{1}$ and Fig. 6). This increase in activation volume of the low resolution scans (Table 1) barely reached statistical significance with paired T-tests uncorrected for multiple comparisons (Table 2) and did not reach statistical significance after correction for multiple comparisons (Table $\mathbf{3}$ ). This lack of statistical significance may be a consequence of the increased dispersion (Table $\mathbf{1}$ and Fig. 6), while activation images at the group level (Fig. 7) show very similar activations in all types of acquisitions. In the second experiment, which is complementary to the first, when GRAPPA was used to achieve high spatial resolution and optimal coverage for whole-brain BOLD fMRI at TR/TE $=3000 / 30 \mathrm{~ms}$ at $3 \mathrm{~T}$, we found no difference in the other metrics. Furthermore, we found consistently higher BOLD values (Table 4), demonstrating that GRAPPA fMRI is useful to gain higher spatial resolution, without detriment to the fMRI measurements.

In this study we showed that acquisition at higher spatial resolution exhibited reduced physiological noise, because the scaling ratio between GRAPPA and non-GRAPPA was very close to what is expected assuming that the noise is thermal in origin. This was not the case for the low-resolution acquisitions where scaling between GRAPPA and non-GRAPPA according to the thermal noise model breaks down, which indicates that physiological noise is a much larger proportion of total noise. Physiological noise is proportional to overall signal $[47,52]$, and so increasing the spatial resolution decreases physiological noise as a proportion of total variance [52]. Although temporal SNR increases with spatial smoothing [53], very broad averaging will "wash out" finer features. Furthermore, all the experimentally calculated tSNR ratios between high resolution acquisitions and low resolution acquisitions also broke the scaling relationships that are expected for thermal noise, which implies that fMRI at higher resolution yields better than expected results. Our findings are in agreement with the literature [29, 47, 52]. Indeed in- creased spatial resolution is beneficial to fMRI because it reduces the contribution of distant veins to the fMRI BOLD signal [37] and thus reduces the point-spread function [36] and BOLD signal displacement [54]. At a voxel size of approximately $8 \mathrm{~mm}^{3}$ at $3 \mathrm{~T}$, the partial voluming effect is reduced and thermal and physiological noise contributions to the tSNR [47] are approximately equal [52]. Such voxel size can be regarded as optimal [55] because lower resolution results in very small gains in tSNR while, in the opposite direction, increased spatial resolution results in decreased SNR. Smaller voxels at higher spatial resolution result in improved voxel homogeneity due to reduced partial voluming effect, and the effective $\mathrm{T} 2 *$ of each voxel more closely corresponds to the $\mathrm{T} 2 *$ of cortical gray matter and white matter, respectively. Larger voxels may suffer extra signal loss since the $\mathrm{T} 2 *$ may be shorter due to the partial voluming effect. Here, we used high spatial resolution GRAPPA to gain improved detection of BOLD signal.

The present study complements and extends previous reports by specifically examining the measured BOLD signal and by providing a double comparison between high and low resolution acquisitions as well as between GRAPPA and non-GRAPPA, performed in two different and complementary experiments. Our finding, in both of our experiments, that measured BOLD values did not decrease in GRAPPA compared to regular (non-GRAPA) fMRI and are higher at higher resolution, demonstrated the utility of GRAPPA fMRI to real clinical applications. In agreement with our results shown in Tables $\mathbf{2}$ and $\mathbf{3}$, previous reports did not find adverse effect of GRAPPA or other pMRI techniques to fMRI. Indeed, we agree with Little et al. who performed a visualfMRI study at 3T and reported that use of GRAPPA did not result in reduced BOLD signals in the occipital cortex, while for several subjects the detected volume was larger when using GRAPPA [28]. We also agree with Preibisch et al. who found no significant reductions in SNR or in statistical power at acceleration factor $R=2$ in a motor-task fMRI study using SENSE (at 1.5T) [29]; we further agree with the results of Preibisch et al. who failed to find any significant reduction in tSNR in a comparison between GRAPPA and non-GRAPPA [26]. Finally, we agree with Lütcke et al. who did not detect any difference between GRAPPA and nonGRAPPA at the same $\mathrm{T} 2 *$-weighting, although their overall assessment of the utility of parallel imaging for fMRI was negative [27].

In conclusion, we purport that use of GRAPPA is advantageous and not detrimental for fMRI at 3T. We therefore suggest the use of GRAPPA to attain higher resolution for fMRI without severely limiting the field of view and to use a TE in the range of $30-40 \mathrm{~ms}$ at $3 \mathrm{~T}$ for optimal BOLD detection. Further improvements in pMRI may result in improved robustness and utility of pMRI for fMRI and other MR applications, and may blur the current distinction between kspace and real-space methods for pMRI [56-58]. Such applications may be ideally suited for the emerging field of preoperative and surgical fMRI [59].

\section{ACKNOWLEDGEMENTS}

This work was supported in part by a grant from the National Institute of Biomedical Imaging and Bioengineering of the National Institutes of Health (Grant number R21 
EB004665-01A2) to A. Aria Tzika. We thank all members of the Athinoula A. Martinos Center for Biomedical Imaging for supporting this work.

\section{REFERENCES}

[1] Zeki S, Watson JD, Lueck CJ, Friston KJ, Kennard C, Frackowiak RS. A direct demonstration of functional specialization in human visual cortex. J Neurosci 1991; 11(3): 641-9.

[2] Fox PT, Mintun MA, Raichle ME, Miezin FM, Allman JM, Van Essen DC. Mapping human visual cortex with positron emission tomography. Nature 1986; 323(6091): 806-9.

[3] Fox PT, Raichle ME. Focal physiological uncoupling of cerebral blood flow and oxidative metabolism during somatosensory stimulation in human subjects. Proc Natl Acad Sci USA 1986; 83(4): 1140-4.

[4] Belliveau JW, Kennedy DN, Jr., McKinstry RC, et al. Functional mapping of the human visual cortex by magnetic resonance imaging. Science 1991; 254(5032): 716-9.

[5] Fox PT, Raichle ME, Mintun MA, Dence C. Nonoxidative glucose consumption during focal physiologic neural activity. Science 1988; 241(4864): 462-4.

[6] Phelps ME, Kuhl DE, Mazziota JC. Metabolic mapping of the brain's response to visual stimulation: studies in humans. Science 1981; 211(4489): 1445-8.

[7] Frostig RD, Lieke EE, Ts'o DY, Grinvald A. Cortical functional architecture and local coupling between neuronal activity and the microcirculation revealed by in vivo high-resolution optical imaging of intrinsic signals. Proc Natl Acad Sci USA 1990; 87(16): 6082-6.

[8] Ogawa S, Tank DW, Menon R, et al. Intrinsic signal changes accompanying sensory stimulation: functional brain mapping with magnetic resonance imaging. Proc Natl Acad Sci USA 1992; 89(13): 5951-5.

[9] Kwong KK, Belliveau JW, Chesler DA, et al. Dynamic magnetic resonance imaging of human brain activity during primary sensory stimulation. Proc Natl Acad Sci USA 1992; 89(12): 5675-9.

[10] Bandettini PA, Wong EC, Hinks RS, Tikofsky RS, Hyde JS. Time course EPI of human brain function during task activation. Magn Reson Med 1992; 25(2): 390-7.

[11] Kim SG, Ashe J, Georgopoulos AP, et al. Functional imaging of human motor cortex at high magnetic field. J Neurophysiol 1993; 69(1): 297-302.

[12] Pruessmann KP, Weiger M, Scheidegger MB, Boesiger P. SENSE: sensitivity encoding for fast MRI. Magn Reson Med 1999; 42(5): 952-62.

[13] Griswold MA, Jakob PM, Heidemann RM, et al. Generalized autocalibrating partially parallel acquisitions (GRAPPA). Magn Reson Med 2002; 47(6): 1202-10.

[14] Griswold MA, Jakob PM, Chen Q, et al. Resolution enhancement in single-shot imaging using simultaneous acquisition of spatial harmonics (SMASH). Magn Reson Med 1999; 41(6): 1236-45.

[15] Moeller S, Van de Moortele PF, Goerke U, Adriany G, Ugurbil K. Application of parallel imaging to fMRI at 7 Tesla utilizing a high 1D reduction factor. Magn Reson Med 2006; 56(1): 118-29.

[16] de Zwart JA, van Gelderen P, Golay X, Ikonomidou VN, Duyn JH. Accelerated parallel imaging for functional imaging of the human brain. NMR Biomed 2006; 19(3): 342-51.

[17] Lupo JM, Banerjee S, Hammond KE, et al. GRAPPA-based susceptibility-weighted imaging of normal volunteers and patients with brain tumor at 7 T. Magn Reson Imaging 2008; 27: 480-8.

[18] Bauer JS, Banerjee S, Henning TD, Krug R, Majumdar S, Link TM. Fast high-spatial-resolution MRI of the ankle with parallel imaging using GRAPPA at 3 T. Am J Roentgenol 2007; 189(1): 2405.

[19] Kim Y, Noll D. Parallel imaging in functional MRI: comparison of spiral SENSE and GRAPPA and of calibration update schemes. Proc Int Soc Magn Reson Med 2006; 14: 2817.

[20] Beatty PJ, Brau AC. Understanding the GRAPPA Paradox. Proc Int Soc Magn Reson Med 2006; 14: 2467.

[21] Clayton D, Skare S, Newbould R, Bammer R. SENSE and GRAPPA Reconstruction of Multi-Shot Multi-Echo EPI Data. Proc Int Soc Magn Reson Med 2006; 14: 2447.
[22] Thunberg P, Zetterberg P. Noise distribution in SENSE- and GRAPPA- reconstructed images: a computer simulation study. Magn Reson Imaging 2007; 25: 1089-94.

[23] Griswold MA, Kannengiesser S, Heidemann RM, Wang J, Jakob PM. Field-of-view limitations in parallel imaging. Magn Reson Med 2004; 52(5): 1118-26.

[24] Ardekani S, Sinha U. Quantitative assessment of parallel acquisition techniques in diffusion tensor imaging at 3.0 Tesla. Conf Proc IEEE Eng Med Biol Soc 2004; 2: 1072-5.

[25] Blaimer M, Breuer F, Mueller M, Heidemann RM, Griswold MA, Jakob PM. SMASH, SENSE, PILS, GRAPPA: how to choose the optimal method. Top Magn Reson Imaging 2004; 15(4): 223-36.

[26] Preibisch C, Wallenhorst T, Heidemann R, Zanella FE, Lanfermann H. Comparison of parallel acquisition techniques generalized autocalibrating partially parallel acquisitions (GRAPPA) and modified sensitivity encoding (mSENSE) in functional MRI (fMRI) at 3T. J Magn Reson Imaging 2008; 27(3): 590-8.

[27] Lutcke H, Merboldt KD, Frahm J. The cost of parallel imaging in functional MRI of the human brain. Magn Reson Imaging 2006; 24(1): $1-5$.

[28] Little MW, Papadaki A, McRobbie DW. An investigation of GRAPPA in conjuction with fMRI of the occipital cortex at 3T. Proc Int Soc Mag Reson Med 2004; 11: 1025.

[29] Preibisch C, Pilatus U, Bunke J, Hoogenraad F, Zanella F, Lanfermann $H$. Functional MRI using sensitivity-encoded echo planar imaging (SENSE-EPI). Neuroimage 2003; 19(2 Pt 1): 412-21.

[30] Brunnstrom S. Motor behavior of adult patients with hemiplegia Movement therapy in hemiplegia. New York: Harper \& Row 1970.

[31] Cramer SC, Nelles G, Schaechter JD, Kaplan JD, Finklestein SP, Rosen BR. A functional MRI study of three motor tasks in the evaluation of stroke recovery. Neurorehabil Neural Repair 2001; 15(1): $1-8$

[32] Khanicheh A, Mintzopoulos D, Weinberg B, Tzika AA, Mavroidis C. MR_CHIROD v.2: magnetic resonance compatible smart hand rehabilitation device for brain imaging. IEEE Trans Neural Syst Rehabil Eng 2008; 16(1): 91-8.

[33] Khanicheh A, Mintzopoulos D, Weinberg B, Tzika AA, Mavroidis C. Evaluation of electrorheological fluid dampers for applications at 3-Tesla MRI environment. IEEE/ASME Trans Mechatron 2008; 13(3): 286-94.

[34] Poldrack RA. Imaging brain plasticity: conceptual and methodological issues--a theoretical review. Neuroimage 2000; 12(1): 1-13.

[35] Parkes LM, Schwarzbach JV, Bouts AA, et al. Quantifying the spatial resolution of the gradient echo and spin echo BOLD response at 3 Tesla. Magn Reson Med 2005; 54(6): 1465-72.

[36] Shmuel A, Yacoub E, Chaimow D, Logothetis NK, Ugurbil K. Spatio-temporal point-spread function of fMRI signal in human gray matter at 7 Tesla. Neuroimage 2007; 35(2): 539-52.

[37] Ugurbil K, Toth L, Kim DS. How accurate is magnetic resonance imaging of brain function? Trends Neurosci 2003; 26(2): 108-14.

[38] Engel SA, Glover GH, Wandell BA. Retinotopic organization in human visual cortex and the spatial precision of functional MRI. Cereb Cortex 1997; 7(2): 181-92.

[39] Jochimsen TH, Norris DG, Mildner T, Moller HE. Quantifying the intra- and extravascular contributions to spin-echo fMRI at $3 \mathrm{~T}$. Magn Reson Med 2004; 52(4): 724-32.

[40] Posse S, Wiese S, Gembris D, et al. Enhancement of BOLDcontrast sensitivity by single-shot multi-echo functional MR imaging. Magn Reson Med 1999; 42(1): 87-97.

[41] Fera F, Yongbi MN, van Gelderen P, Frank JA, Mattay VS, Duyn JH. EPI-BOLD fMRI of human motor cortex at $1.5 \mathrm{~T}$ and $3.0 \mathrm{~T}$ : sensitivity dependence on echo time and acquisition bandwidth. J Magn Reson Imaging 2004; 19(1): 19-26.

[42] Wansapura JP, Holland SK, Dunn RS, Ball WS, Jr. NMR relaxation times in the human brain at 3.0 tesla. J Magn Reson Imaging 1999; 9(4): 531-8.

[43] Firbank MJ, Coulthard A, Harrison RM, Williams ED. A comparison of two methods for measuring the signal to noise ratio on MR images. Phys Med Biol 1999; 44(12): N261-4.

[44] Haacke ME, Brown RW, Thompson MR, Ramesh V. Magnetic resonance imaging: physical principles and sequence design. New York: Wiley 1999.

[45] Dietrich O, Raya JG, Reeder SB, Ingrisch M, Reiser MF, Schoenberg SO. Influence of multichannel combination, parallel imaging and other reconstruction techniques on MRI noise characteristics. Magn Reson Imaging 2008; 26(6): 754-62. 
[46] Dietrich O, Raya JG, Reeder SB, Reiser MF, Schoenberg SO. Measurement of signal-to-noise ratios in MR images: influence of multichannel coils, parallel imaging, and reconstruction filters. J Magn Reson Imaging 2007; 26(2): 375-85.

[47] Kruger G, Glover GH. Physiological noise in oxygenation-sensitive magnetic resonance imaging. Magn Reson Med 2001; 46(4): 631-7.

[48] Liu CS, Miki A, Hulvershorn J, et al. Spatial and temporal characteristics of physiological noise in fMRI at 3T. Acad Radiol 2006; 13(3): 313-23

[49] Friston KJ, Holmes A, Poline JB, Price CJ, Frith CD. Detecting activations in PET and fMRI: levels of inference and power. Neuroimage 1996; 4(3 Pt 1): 223-35.

[50] Gudbjartsson H, Patz S. The Rician distribution of noisy MRI data. Magn Reson Med 1995; 34(6): 910-4.

[51] Constantinides CD, Atalar E, McVeigh ER. Signal-to-noise measurements in magnitude images from NMR phased arrays. Magn Reson Med 1997; 38(5): 852-7.

[52] Triantafyllou C, Hoge RD, Krueger G, et al. Comparison of physiological noise at $1.5 \mathrm{~T}, 3 \mathrm{~T}$ and $7 \mathrm{~T}$ and optimization of fMRI acquisition parameters. Neuroimage 2005; 26(1): 243-50.

[53] Triantafyllou C, Hoge RD, Wald LL. Effect of spatial smoothing on physiological noise in high-resolution fMRI. Neuroimage 2006; 32(2): 551-7.
[54] Olman CA, Inati S, Heeger DJ. The effect of large veins on spatial localization with GE BOLD at $3 \mathrm{~T}$ : displacement, not blurring. Neuroimage 2007; 34(3): 1126-35.

[55] Bodurka J, Ye F, Petridou N, Murphy K, Bandettini PA. Mapping the MRI voxel volume in which thermal noise matches physiological noise--implications for fMRI. Neuroimage 2007; 34(2): 542-9.

[56] Blaimer M, Breuer FA, Seiberlich N, et al. Accelerated volumetric MRI with a SENSE/GRAPPA combination. J Magn Reson Imaging 2006; 24(2): 444-50.

[57] Griswold MA, Breuer F, Blaimer M, et al. Autocalibrated coil sensitivity estimation for parallel imaging. NMR Biomed 2006; 19(3): 316-24.

[58] Griswold MA, Blaimer M, Breuer F, Heidemann RM, Mueller M, Jakob PM. Parallel magnetic resonance imaging using the GRAPPA operator formalism. Magn Reson Med 2005; 54(6): 1553-6.

[59] Liu WC, Feldman SC, Schulder M, et al. The effect of tumour type and distance on activation in the motor cortex. Neuroradiology 2005; 47(11): 813-9.

(C) Mintzopoulos et al.; Licensee Bentham Open.

This is an open access article licensed under the terms of the Creative Commons Attribution Non-Commercial License (http://creativecommons.org/licenses/by-nc/3.0/) which permits unrestricted, non-commercial use, distribution and reproduction in any medium, provided the work is properly cited. 